\title{
Smart Modular Dispenser for Medication Administration
}

\author{
Ana Rita Sousa1, Pedro Dinis Gaspar ${ }^{1,2}$ \\ ${ }^{1}$ University of Beira Interior \\ Rua Marquês d'Ávila e Bolama, 6201-001, Covilhã, Portugal \\ ana.rita.nunes.sousa@ubi.pt; dinis@ubi.pt \\ ${ }^{2} \mathrm{C}-\mathrm{MAST}$ - Centre for Mechanical and Aerospace Science and Technologies \\ Covilhã, Portugal
}

\begin{abstract}
Over the years, average life expectancy tends to increase, which directly translates into an increase of the aging population. Consequences of this process are the decline or loss of cognitive and motor capacities, reflecting directly on the quality of life and the autonomy of the people, compromising the fulfillment of a pharmacological plan. It is known that, inevitably, medicines are increasingly introduced as part of human life in order to improve the quality of life. However, especially in the elderly and people with dementia, the difficulties in strictly complying with their medicine intake are increasingly evident. In order to counteract this situation, this article presents a device that assists them in this challenge, overcoming situations of overdose, drug interactions and even forgetfulness, due to the lack of recall of instructions, doses and schedules. Although there are different types of pills dispensers on the market, the disclosure herein includes a number of novel features. It is a small and portable pill dispenser so that it is easy to carry. It is able to adapt to the pharmacological needs of the individual since it is modular. It presents audible and luminous alerts in order to facilitate their use and interpretation. This device is mostly used for pills of various shapes and sizes, but is also intended to be capable of holding drops or powdered medication.
\end{abstract}

Keywords: Pill dispenser, Modular, Smart, Medication, Alarm, Pharmacological Plan.

\section{Introduction}

Over the years, the average life expectancy tends to increase, resulting in an increase of the aging population. This process of aging is followed by a decrease or loss of cognitive and motor abilities, altering the quality of life and the autonomy of the individual, namely with respect to the fulfillment of its pharmacological plan [1].

The human being, in order to improve and prolong their quality of life, ends up consuming a high number of medicines. The increase in drug consumption is notorious in all age groups, but the elderly present a greater enhancement. In older people, a greater number of medicines and a greater complexity of the pharmacological plan are observed compared to the other age groups, as they usually need to take different types of medicines at different doses and times. Since in the elderly there is a high decrease or loss of cognitive, visual and auditory capacities and a degradation of the memory capacity, they present difficulties in reading labels, inability to remember instructions, doses and schedules, which causes errors in the correct intakes of your medication. All of these aspects can lead to serious errors in the health of the individual, since overdose, drug interactions and even forgetfulness can occur, resulting in an ineffective therapy and, consequently, can lead to serious health conditions that may lead to hospitalization or even in severe cases to death [2] [3].

Another population group to consider are individuals with dementia, since they present a decline in mental functions, which interferes with the normal functioning of the individual, and, like the elderly, they are unable to comply rigorously and correctly with their pharmacological plan because of its limitations.

In view of all the problems mentioned above, it is understood that it is necessary to create a simple and intelligent device, to help patients remember instructions, doses and schedules and to alert them when taking their medicines, thus avoiding errors in the compliance of their pharmacological plan [4]. 


\section{Literature Review}

Currently there is a wide range of devices that help patients in their medication shots and help to comply with the pharmacological plan. These devices can be simpler, such as tablet organizers, to more complex devices, composed of intelligent systems with audible and luminous alerts [5].

The pillboxes organizer essentially serves to organize medication takes according to when they have to be taken. It is responsibility of the patient to manage the time at which the medication compartment opens. The situation compromises the use of these devices in a variety of situations, since human failure is very likely, especially in older people.

With the evolution of technology, particularly in the area of health, smart dispensers have been created. These devices are an added value for the patient since they are configurable: it is possible to unambiguously define the times of the medication taking, emitting sound and luminous alerts whose purpose is to assist the patient in the execution of the correct intake, avoiding forgetfulness that causes deviations in the prescribed intake hours. In devices with associated software, greater control over the patient's activity is also possible, and it is up to the caregiver to analyse the data generated by the information system and take the measures that best suit the patient health. Thus, intelligent dispensers are an added value, facilitating the life of the caregivers and helping to comply with the pharmacological plan of the patients, contributing, in this way, and indirectly, to an improvement of the quality of life and greater safety in the treatment of the elderly and of patients with special needs. These devices can also improve society in general and health systems by reducing the need for the presence of health professionals, being possible to focus more on new clinical cases, that consequently leads to a decrease in waiting times and costs, aspects that are critical in the treatment of urgent cases [6] [7].

Several studies have been conducted that address and discuss the efficacy and limitations of dispensing devices. The study performed by Mertens et al. [8] in 44 community pharmacies in the Netherlands aimed to compare medication management problems in patients using multidose drug dispensing (MDD) systems and patients who dispensed medication manually. 440 patients, 65 years of age or older, staying at home, and that consume a minimum of five chronic prescribed medications participated in the study. A questionnaire was applied covering four fields: functional, organizational, adherence and medication problems. This survey served for health professionals to identify patients with reduced medication management capacity. It was found that patients using MDD systems were older, consumed more medication, were cognitively more debilitated, and presented more medication management and handling problems compared to patients who did not use such systems. Although MDD systems present higher costs than manual dispensing, they are able to bridge failures in the ability of some patients to correctly identify medication and not be able to adhere to a complex pharmacological plan [8].

Reeder et al. [9] indicate that the National Institute of Health of the USA submitted a questionnaire to the satisfaction of 96 elderly people with at least 60 years old, having home health care and who, although weak, have the ability to follow simple instructions autonomously. They used MD. 2 dispensers continuously for 12 months. The objective was to draw conclusions regarding ease of use, reliability, management assistance with medication, improvement of routine tasks and acceptance of the method, aspects that were evaluated with a score of 1 to 5 . The results obtained were unanimous. All five aspects under analysis obtained a maximum score in at least $90 \%$ of the patients, showing that, effectively, the use of devices was simple and that they were an added value for their drug management. Some patients have also reported that the use of the device did not interfere with their independence of movement and that, although it is initially not easy to accept the device, adaptation to it is progressively performed successfully. $84 \%$ of patients showed willingness to use the device in the future [9].

Sinnemäki et al. [10] reviewed several studies in order to draw general conclusions about the use and applicability of medical dispensers. A series of conditions were formulated that would have to be satisfied in order to validate the studies. This review contained 328 studies, however only 7 studies were considered valid and of these only 4 had control group. It was concluded that there is still insufficient information to support unequivocally the automatic dispensers influence on appropriateness and safety of medication use and costs, however, studies that have been validated maintain that these devices can reduce errors of medication intake, improving the consistency of medication and, consequently, assisting patients. They also state the trend of these devices to be only used by the more fragile population and, consequently, more prone to the conception of errors in the medication doses. It has been reported that devices may promote the overly prolonged medication of a treatment or a dose when compared to manual devices if the patient is not closely followed by the caregiver [10]. 
The study developed by Nakrem et al. [11] reveals that the use of technology in health can undermine the patientcaregiver relationship. Despite facilitating patients' independence by making them more autonomous, it leads to an increase in the concerns of health professionals regarding the safety of the use of automatic dispensers in dependent patients. It is necessary to ensure that the actions of health professionals are correctly replaced by the device, and control and monitoring by health professionals is also required [11]. According to a study conducted by Cheung et al. [12], in a sample of 15,113 incidents in medication intakes, 268 were associated with taking with automated dose dispensing (ADD) service. Most of these errors arise in the change of the pharmacological plans of the patient. These errors were generally associated with human failures when inserting the data in the information system or in the replacement of medicines. However, the percentage of errors made by the smart dispensers is reduced. This value justifies a relatively safe method. However, it should be noted that the percentage of patients who opt for the ADD medication is not known.

According to the Canadian Agency for Drugs and Technologies in Health, in a survey of 78 Canadian hospitals, 56\% use some type of automatic drug dispenser and 33\% say that automatic dispensers will be the next investment. In another more recent survey it is portrayed that $75 \%$ in Canada and $83 \%$ in the United States would already be using automatic dosing systems. The increasing adoption of these systems in the real environment is a very strong indicator that reveals benefits in its use, in terms of reducing costs and increasing hospital efficiency as an improvement in the clinical treatment process [13].

Given the enormous diversity of existing devices, only the most relevant, sophisticated devices with an intelligent system are targeted for this work. The E-pill MedSmart Automatic Pill Dispenser [14] consists of a portable circular device with 29 compartments, of which 28 are used for drug storage. Each compartment of this dispenser has a capacity for twenty pills the size of an aspirin. It has audible and luminous alerts and a screen that allows you to view a series of information relevant to the user, such as the current date and time, the number of daily alarms and the time of the next alarm. When the alarm is activated, the respective compartment is accessible and, in order for the patient to have access to the pills, the device must be tilted. This dispenser has already received updates, in which it has been implemented the possibility of the caregiver being notified via phone call, email or mobile phone message to remotely monitor the therapeutic plan and alert you in the event of any failure. The Philips Automated Medication Dispensing Service [15] and Hero [16] are larger devices and therefore not easily transportable. The first is composed of a set of ten cylinders with six plastic containers in which the pills are placed, in an orderly manner by the time of the takes. The second has ten containers to place the pills. Both feature a bright, audible display, buttons, and alerts. When the alarms are activated, the user must press the button next to the screen that provides access to the container with the pills. MedTracker [17] is a system that records all the movements of medicines made by the user in a dispenser. Subsequently, a communication with a computer is made via bluetooth in which it is possible to extract statistics and relevant data regarding the consumption of different kind of medications. This system, however, serves only as a complement to dispensers, since it does not implement any type of dispensing control mechanism or any type of alerts. Buono et al. [18] have developed a modular drug dispenser consisting of a base that serves as a support and feeding to a set of pillboxes and provide several alerts that warn the patient of the time of taking the medication. This device has been developed with the aim of providing a home treatment orientation for the patient, being indicated for patients dependent on third parties. Despite being modular and theoretically presenting an unlimited capacity to support pillboxes, it is not easily transportable. However, it allows taking several types of drugs. The control and configuration of the medicated pills are performed by a smartphone application, by the caregiver. This device is intended for use at home.

It is found that existing smart dispensers have some advantages in aiding the compliance of the patient's pharmacological plan, but they do have some disadvantages. When they are small and portable, they have limitations on the amount of medications that their compartments can house. When devices feature larger compartments and more sophisticated alert systems, they are large in size and therefore not easy to carry.

Thus, given the different limitations in each of the devices, the opportunity to create a complete device, robust and simple to use and to carry arose.

\section{Device Description}

The Smart Modular Dispenser for Medication Administration is a small, portable, smart medication dispenser made up of modules. These modules, in the standard form of the device, are parallelepiped and approximate dimensions $7 \times 5 \times 2.5 \mathrm{~cm}$, making a total of $20 \mathrm{~cm}$ through the maximum limit of modules. Thus, it has a smaller size than other competing devices on the market. This device has a maximum limit of eight modules, one of which is the control module, due to transport easiness specification. 
One of the characteristics that distinguishes it from other existing devices is modularity, given the possibility of the user adapting the number of modules by the number of pills that make up his pharmacological plan. Each module corresponds to one type of drug, i.e. a patient taking two different medicaments will only need one device with three modules correspondingly. One patient presenting a pharmacological plan consisting of five different medicaments will need a device with six modules.

The control module (1) has a microcontroller that allows connection to the remaining modules, a timer to help set the time for taking the medicine, a battery for a power supply, a red LED (2) that indicates low battery, a buzzer that emits sound alerts and a network card that provides a connection, via wi-fi or other means of communication, with control panel (browser) that allows to configure all parameters according to the pharmacological plan the information stored in the database. This database is used for the management and storage of data, such as the personal data of the user, history of diseases and the prescriptions prescribed by the doctor.

The configuration of the parameters of the drug intake will be made by the administrator (doctor or pharmacist), allowing to establish a patient-medical-pharmaceutical network. The doctor issues a prescription that is stored in the database, later the pharmacist accesses this prescription, being responsible for setting the parameters of the device through the pharmacological plan of the patient and loading it with the corresponding medicines. The patient only needs to go to the pharmacy to pick up the device, follow the instructions given by the device's smart system and go to the pharmacy to recharge it on demand. It should be noted that only the administrator is able to access the control panel and configure the parameters of the drug intake, as it has a password that should not be transmitted to the user.

A diagram of the device is shown in Figure 1. Figure 2 shows a diagram of the interior of one of the dispensing modules.

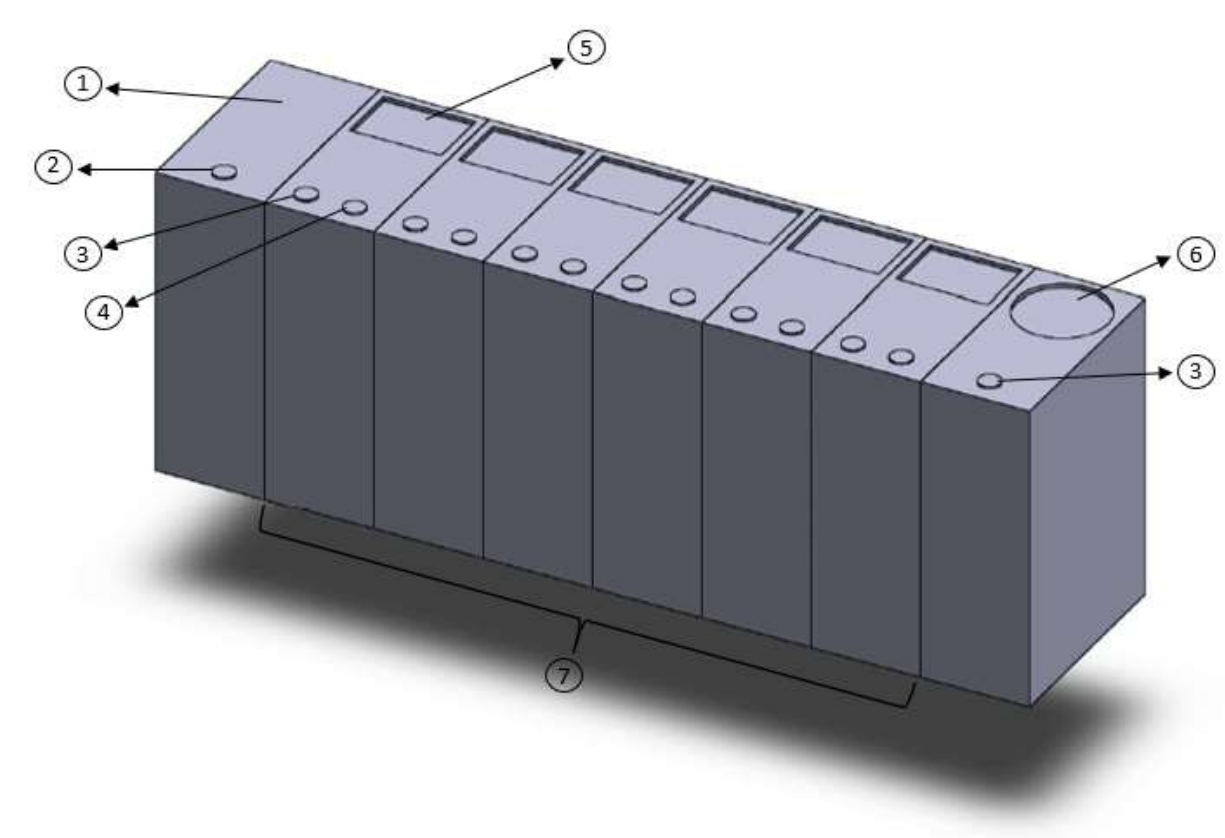

Fig. 1: Schematic example of the Smart Modular Dispenser for Medication Administration. 


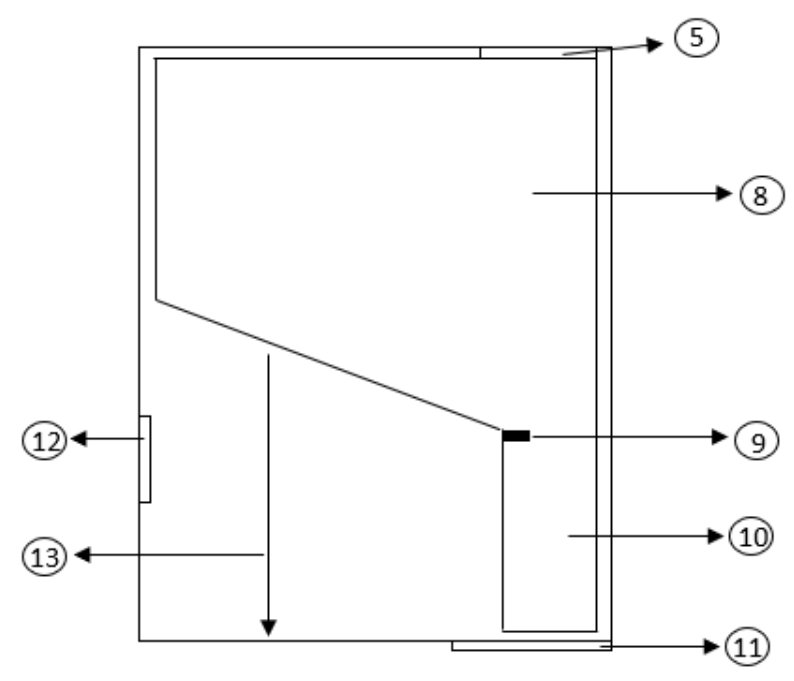

Fig. 2: Diagram of the interior of a dispenser module.

Each of the remaining modules (7) has a simple design whose purpose is to facilitate the patient use. Accordingly, each of the modules therein has a compartment (8) where the pills are stored; a column (10) through which the tablets leave; a brake (9) which prevents the extraction of more than one each at a time and an actuation system, which is operated by a mechanical system (spring) (13), and there is also the possibility of operating through an electromechanical system. On the outside of the module, at the top, there is a button (5) which, as soon as the alerts are issued, is easily pressed by the patient. There are also two LEDs, one green (3) and one red (4), which respectively indicate the module in which the patient should press the button and indicate when the module needs recharging. There is a minimum number of pills stored. Each module should never be completely empty so that there is no failure to comply with the patient's pharmacological plan.

In order to control the number of pills that are removed, each of the modules, with the exception of the control module and the adapter module, it has a contact sensor (12) on one of its inner sides, so that, when the button is pressed, the compartment housing the pills come down and touch that sensor, giving indications to the device that a pill was dispensed. This feature is useful because when the patient has to take two equal tablets at the same time, it is possible to count the number of pills that have been removed from the module in order to turn off the alerts as soon as the medication dose is taken. For patient safety, at the base of each module, in order to prevent drug delivery, there is an enclosed electromagnet (11). It only opens when the set time is reached, and the button corresponding to the module is pressed, issuing the alert which, prevents the patient from making mistakes in the intakes.

One of the innovative features of this device is the existence of a module adapted for powdered or droplet medication (6), i.e. it is possible to integrate in the device a module which has a cushioned cylinder into which is inserted a medication bottle in drops or a cylinder with a capacity of about $30 \mathrm{ml}$ (approximate volume determined by calculating the volume of the cylinder) used to pour water and mix with the powdered medication. This module, however, only serves as a support and allows to alert when it is the time of its administration, not having implemented mechanisms of accounting of the doses. It has a green LED (3) that indicates when it is time to give the drops or powder.

In short, the main features of this device are modularity, intelligent system, portability, easy to carry, simple interface and design, easily interpreted and used, sound and luminous alerts, superior capacity to host tablets of various shapes and sizes and has a module adapted for drops and powder medication. All these factors make this device a product with enough potential, which fills the flaws of existing products for this purpose.

\section{Conclusion}

Increasingly, aging is part of our reality, which is good in the sense that people may live longer, but there is a greater complexity in the treatment of patients. As the medicaments are usually used for the cure and treatment of several pathologies, an increase in their consumption is observed in order to improve the longevity and quality of life of the people. Faced with 
the limitations in compliance to the pharmacological plan, especially in the elderly and those with dementia, the need for the creation of a device to assist them arose.

Although there is a wide range of devices designed to assist patients in complying with their pharmacological plan, they have some limitations. Therefore, this Smart Modular Dispenser for Medication Administration comes with the intention of attempting to address these individual failures. This device presents a set of features that, until then, are not found in any other existing device. The main distinguishing features of this device are its modularity, and the patient can adapt the number of modules by the number of medicines he takes. Each module is capable of harboring a greater number of tablets of various shapes and sizes and is capable of alerting to powdered and droplet medication since it may couple a carrier for such an effect. All the mentioned characteristics, coupled in a single device, make of this a device with great potential for the market.

\section{Future Enhancements}

With regard to future improvements, the constant and gradual monitoring of technology is necessary for the device to be competitive, fully functional and current. It would be helpful and would allow greater control on the part of the caregivers if a mobile application could be coupled to this device in which the caregiver would have access to information such as weekly recording of medication intakes and missed doses, an alert to the caregiver when doses were not taken. It is open to a future possibility of implementing an alternative functionality so that a trustworthy caregiver is able to configure the parameters of the medication intake through an application facilitating the work of the administrator (doctor or pharmacist) and simplifying the network between patient, pharmacist and physician. Another important aspect is that this device presents sound and luminous alerts, however, if the user's profession does not allow the sound alert to be activated, it would be good if this sound alert could be replaced by a vibrating alert. Finally, although a simple and appealing design has been chosen, it can always be optimized in order to contribute to the user's comfort and well-being.

\section{References}

[1] WHO, "Study on global AGEing and adult health", World Health Organization (WHO), Geneva, 2015.

[2] A. Naditz, "Medication Compliance - Helping Patients Through Techonology: Modern "Smart" Pillboxes Keep Memory - Short Patients On Their Medical Regimen," Telemedicine and e-Health, vol. 14, no. 9, pp. 875, 2008.

[3] V. Pepe and C. Castro, "A Interação entre prescritores, dispensadores e pacientes: informação compartilhada como possível benefício terapêutico," in Cad. Saúde Pública, Rio de Janeiro, vol. 16, no. 3, pp. 815-822, 2000.

[4] P. Cheek, L. Nikpour and H. D. Nowlin, "Aging Well With Smart Techonology," Nursing administration quarterly, vol. 29 , no. 4, pp. 329-38, 2005.

[5] S. Mukund and N.K.Srinath, "Design of Automatic Medication Dispenser," Computer Science \& Information Technology, 2012.

[6] J.-H. Do, S. H. Jung, H. Jang, S.-E. Yang, J.-W. Jung and Z. Bien, "Gesture-Based Interface for Home Appliance Control in Smart Home," Assistive Technology Research Series 19: Smart Homes and Beyond, IOS Press, 2006.

[7] S. R. B. Shree, P. C. Shekar, A. Arjun, G. R. Manoj, A. Nithin and R. S Raj, "Automated Medication Dispensing System," 2014 Eleventh International Conference on Wireless and Optical Communications Networks (WOCN), IEEE, 2014.

[8] B. J. Mertens, H. F. Kwint, R. J. van Marum, and M. L. Bouvy, "Are multidose drug dispensing systems initiated for the appropriate patients?”, Eur J Clin Pharmacol., vol. 74, no. 9, pp. 1159-1164, 2018.

[9] B. Reeder, G. Demiris, and K. D. Marek, "Older Adults' Satisfaction with a Medication Dispensing Device in Home Care,” Inform Health Soc Care., vol. 38, no. 3, pp. 211-222, 2013.

[10] J. Sinnemäki, S. Sihvo, J. Isojärvi, M. Blom, M. Airaksinen, and A. Mäntylä, “Automated dose dispensing service for primary healthcare patients: a systematic review," Syst Rev., vol. 2, no. 1, 2013.

[11] S. Nakrem, M. Solbjør, I. N. Pettersen, and H. H. Kleiven, "Care relationships at stake? Home healthcare professionals' experiences with digital medicine dispensers - a qualitative study," BMC Health Serv Res., vol. 18, pp. 26, 2018.

[12] Ka-Chun Cheung, Patricia M. L. A. van den Bemt, Marcel L. Bouvy, Michel Wensing, and Peter A. G. M. De Smet, "Medication Incidents Related to Automated Dose Dispensing in Community Pharmacies and Hospitals - A Reporting System Study," PLoS One., vol. 9, no. 7, pp. e101686, 2014. 
[13] Health Technology Inquiry Service, "Automated Medication Dispensing Systems: A Review of the Clinical Benefits, Harms, and Cost-Effectiveness," Health Technology Assessment. 30 Sep. 2010.

[14] E-pill. (n. d). e-pill MedSmart Automatic Pill Dispenser with Patient Compliance Dashboard. [Online]. Available: https://www.epill.com/medsmart.html

[15] Philips. (n. d). Automated Medication Dispensing Service. [Online]. Available: https://www.lifeline.philips.com/business/medicationdispensing

[16] Herohealth. (n. d). Hero. [Online]. Available: https://herohealth.com/

[17] T.L. Hayes, J.M. Hunt, A. Adami and J.A. Kaye, "An electronic pillbox for continuous monitoring of medication adherence," 28th IEEE EMBS Annual International Conference, Aug. 30-Sept. 3, 2006.

[18] P. Buono, F. Cassano, A. Legretto and A. Piccinno, "A Modular Pill Dispenser Supporting Therapies at Home". Current Trends in Web Engineering: ICWE 2018 International Workshops. C. Pautasso, F. Sánchez-Figueroa, K. Systä, J. M. M. Rodríguez (Eds). LNCS 11153, Springer, 2018. 